\title{
Oncoxin nutritional supplement in the management of chemotherapy- and/or radiotherapy-associated oral mucositis
}

\author{
ALEXANDR SHUMSKY $^{1}$, EVGENIY BILAN ${ }^{2}$, EDUARDO SANZ $^{3}$ and FEDOR PETROVSKIY ${ }^{4}$ \\ ${ }^{1}$ Medical Scientific Centre of Professor Shumsky, 443303 Samara; ${ }^{2}$ Khanty-Mansiysk Regional Hospital, \\ 628011 Khanty-Mansiysk, Russia; ${ }^{3}$ Catalysis S.L., 28016 Madrid, Spain; \\ ${ }^{4}$ Khanty-Mansiysk State Medical Academy, 628011 Khanty-Mansiysk, Russia
}

Received July 13, 2018; Accepted February 6, 2019

DOI: $10.3892 / \mathrm{mco} .2019 .1809$

\begin{abstract}
Oral mucositis (OM) is a common and potentially dangerous complication of anticancer treatment. Since there are only few therapeutic options for OM, there is a need to identify novel approaches to its management and prevention. The aim of the present study was to evaluate the efficacy of Oncoxin (ONCX), a nutritional supplement that contains microelements, vitamins, amino acids and certain biologically active substances of natural origin, in cancer patients who receive chemotherapy, radiotherapy or a combination of the two. A total of 15 male and female patients (aged 45-75 years) with malignant neoplasms, who had been prescribed radiotherapy, chemotherapy or a combination of the two, with an Eastern Cooperative Oncology Group performance status score $\leq 3$, and grade 2-3 OM based on the World Health Organization (WHO) Oral Toxicity Scale, were enrolled in a 20-day study; 10 patients were in the ONCX group and 5 served as controls. The patients were allowed to use any anticancer treatment and any type of OM care. In addition to their current treatment, patients in the ONCX group used $25 \mathrm{ml}$ of the ONCX nutritional supplement twice daily for 20 days. The mean WHO Oral Toxicity Scale grade decreased by $41 \%$ in the ONCX group after $\sim 7$ days from the beginning of the study compared with minimal change in the control group. At the end of the study, the difference was even more prominent, with a 73 and $20 \%$ decrease from baseline in the ONCX and control groups, respectively $(\mathrm{P}<0.001)$. During the entire trial period, patients in the ONCX group were able to eat normally during $65 \%$ of the time, in contrast to only $29 \%$ in the control group $(\mathrm{P}=0.04)$. There were no statistically significant changes
\end{abstract}

Correspondence to: Professor Fedor Petrovskiy, Khanty-Mansiysk State Medical Academy, ul. Mira 40, 628011 Khanty-Mansiysk, Russia

E-mail: fedor_petrovsky@mail.ru

Abbreviations: OM, oral mucositis; ONCX, Oncoxin

Key words: oncoxin, oral mucositis, chemotherapy, radiotherapy in absolute body mass, or in the number of days with normal appetite. This was a pilot study aiming to show the benefits of the ONCX nutritional supplement in OM, and the results demonstrated that ONCX rapidly improved the symptoms of $\mathrm{OM}$ and helped to maintain normal eating habits in patients undergoing cancer treatment.

\section{Introduction}

Oral mucositis $(\mathrm{OM})$ is a common and potentially dangerous complication of anticancer treatment. Inflammation and ulcerations of the oral mucosa make swallowing and, thereby, eating and drinking, difficult or even impossible, leading to anorexia and weight loss (1). There is a serious risk of infections and even sepsis, particularly in immunocompromised patients $(2,3)$. OM is associated with severe pain, affects the quality of life, and often requires reduction or suspension of chemotherapy and/or radiotherapy, thereby decreasing the relative dose intensity, which may worsen prognosis $(2,3)$.

The risk and severity of mucositis depend on the type of therapy. It is diagnosed in $60-100 \%$ of patients receiving myeloablative therapy and in almost all patients on combined chemotherapy and radiotherapy $(1,4)$. OM initially manifests with erythematous spots on the oral mucosa or tongue, which subsequently develop into painful ulcers $(1,2)$. Xerostomia is common and represents one of the most troublesome symptoms of OM $(1,2)$. Ulcers, xerostomia and restricted diet lead to microbiome disruption and colonization by opportunistic or pathogenic microorganisms (2). Dental caries, periodontal disease, ill-fitting dentures and other such problems must be resolved prior to the initiation of anticancer therapy and further treatment as needed (1). Poor oral hygiene, smoking and alcohol use exacerbate OM symptoms and trigger oral infections (2). Approximately 1 in 10 patients on radiotherapy or chemoradiotherapy will develop severe $\mathrm{OM}$ and may require hospitalization and treatment rescheduling (2).

The first clinical signs of OM usually appear 4-5 days after initiation of chemotherapy, with development of ulcerations after 7-10 days; the ulcers tend to grow in number and size and merge to form large ulcerated areas $(2,3)$. These ulcers are very painful, cause difficulty in swallowing and may necessitate the administration of opioid analgesics. They require $\sim 2$ weeks to heal once chemotherapy has been suspended $(2,3)$. 
Since there are currently few therapeutic options for OM, there is a need to identify novel approaches to its management and prevention. Oncoxin (ONCX), a nutritional supplement enriched with amino acids, vitamins, minerals and naturally occurring antioxidants, has been previously demonstrated to improve appetite, quality of life and overall survival in patients with end-stage hepatocellular carcinoma (5). The aim of the present study was to evaluate the efficacy of ONCX in cancer patients with OM who receive chemotherapy, radiotherapy or a combination of the two.

\section{Materials and methods}

Study population. The eligibility criteria for inclusion in the study were as follows: Male and female patients who had signed an informed consent, aged 45-75 years, with malignant neoplasms and who had been prescribed radiotherapy, chemotherapy or a combination of the two, with an Eastern Cooperative Oncology Group performance status score of $\leq 3$, with grade 2-3 OM according to the World Health Organization (WHO) Oral Toxicity Scale. The exclusion criteria were as follows: Severe concomitant diseases or conditions that may complicate or make impossible the patient's participation in the study, or make it difficult to interpret the clinical data (including mental disorders, severe infectious and parasitic diseases and intolerability to any of the ONCX components), the patient's family or official relations with a member of staff of the study center, the patient's failure to assess his/her physical and/or emotional condition, the patient's failure to comply with the study requirements, the patient's refusal to participate in the study and pregnancy or lactation.

The present study was approved by the Ethics Committee of The Loginov Moscow Clinical Scientific Centre, protocol 3/2017 4.17.17. The inclusion period was between November 2017 and March 2018. In accordance with the Declaration of Helsinki, all patients provided written informed consent to participate in the study and to publish the results and anonymized photos. The study was retrospectively registered under the study registration number NCT03577535.

Study design and treatment. The present study was a pilot study conducted to verify the hypothesis that ONCX can improve the symptoms of OM. The primary objective of the study was to evaluate the efficacy of ONCX in patients with grade 2-3 OM based on the WHO Oral Toxicity Scale. The following outcome measures were used: Mean WHO Oral Toxicity Scale grade (primary endpoint), body mass, days with regular food intake, days with normal appetite, adverse events and the Common Toxicity Criteria (ver. 2) of the National Cancer Institute (https://ctep.cancer.gov/protocoldevelopment/electronic_applications/docs/ctcv20_4-30-992. pdf) using the blood, hepatic and infection scales. The study regimen is shown in Fig. 1. This study was a single-center, open-label, randomized trial in two parallel groups with a 20-day treatment period. No follow-up period was intended. The study was conducted at a stomatological clinic (Medical Scientific Centre of Professor Shumsky, Samara, Russian Federation). Patients were randomly allocated in 2:1 comparison groups for the ONCX and control groups, respectively. A total of 15 patients were enrolled in the study; 10 in the ONCX
Table I. Composition of Oncoxin per $100 \mathrm{ml}$.

\begin{tabular}{lr}
\hline Components & Quantity \\
\hline Glycine & $2,000 \mathrm{mg}$ \\
Glucosamine & $2,000 \mathrm{mg}$ \\
Malic acid & $1,200 \mathrm{mg}$ \\
Arginine & $640 \mathrm{mg}$ \\
Cysteine & $204 \mathrm{mg}$ \\
Mono-ammonium glycyrrhizinate & $200 \mathrm{mg}$ \\
Ascorbic acid & $200 \mathrm{mg}$ \\
Sodium methylparaben & $120 \mathrm{mg}$ \\
Zinc sulfate & $100 \mathrm{mg}$ \\
Green tea extract & $80 \mathrm{mg}$ \\
Calcium pantothenate & $25 \mathrm{mg}$ \\
Pyridoxine & $12 \mathrm{mg}$ \\
Manganese sulfate & $4 \mathrm{mg}$ \\
Cinnamon extract & $3 \mathrm{mg}$ \\
Folic acid & $400 \mu \mathrm{g}$ \\
Cyanocobalamin & $2 \mu \mathrm{g}$ \\
\hline
\end{tabular}

group and 5 as controls. Sequentially numbered opaque sealed envelopes were used as the method for randomization. The study center was provided with excess amount of ONCX and excess number of envelopes.

Treatment. Patients from both groups were allowed to use any anticancer treatment and any OM care prior to the present study. In addition to their current treatment, patients in the ONCX group used $25 \mathrm{ml}$ of the ONCX nutritional supplement twice daily for 20 days. ONCX contains microelements, vitamins, amino acids and certain naturally occurring, biologically active substances; the composition of ONCX is presented in Table I. The next course of chemotherapy or chemoradiotherapy was withheld until the full resolution of OM symptoms. Oral cavity and dental treatment were provided to all patients as the standard procedure before the start of chemotherapy. Caries and dental deposits were treated if necessary during the study. As a part of oral care, patients used oral washes containing antiseptics including octenidine dihydrochloride and phenoxyethanol or myramistin. To accelerate the regeneration process, Solcoseryl dental adhesive paste and Actovegin tablets were used. Patients were advised to use soft or very soft toothbrushes and non-abrasive toothpaste with a foaming effect. In case of moderate or severe pain, patients were advised to use nimesulide tablets and local application of lidocaine gel.

Statistical analysis. Statistical analysis was performed using StatSoft Statistica 10.0 software (http://statsoft.ru/). Baseline characteristics are presented as mean \pm standard deviation; when comparisons between groups or within a group were made, the data were presented as mean (95\% confidence interval). The differences between the ONCX and control groups were compared using Mann-Whitney $\mathrm{U}$ test and the differences within each group were compared using sign test; $\mathrm{P}<0.05$ was considered to indicate a statistically significant difference. 
Group 1. Anticancer treatment + oral mucositis therapy + ONCOXIN 25 ml twice daily (ONCX group)

\begin{tabular}{|l|l|}
\hline \multicolumn{2}{|l|}{ Group 2. Anticancer treatment + oral mucositis therapy (control group) } \\
\hline Visit 1 & Visit 2 \\
\end{tabular}

Figure 1. Study regimen. A total of 3 visits were scheduled. Visit 1 took place on the day when the expression of oral mucositis symptoms on the WHO Oral Toxicity Scale reached a grade of 2-3; visit 2 was scheduled 6-8 days after Visit 1 and Visit 3 was scheduled 19-21 days after Visit 1.

Table II. Patient baseline characteristics according to group allocation.

Groups (mean \pm standard deviation)

\begin{tabular}{lcc}
\cline { 2 - 3 } Characteristics & Oncoxin $(\mathrm{n}=10)$ & Control $(\mathrm{n}=5)$ \\
\hline Age, years & $62.0 \pm 7.9$ & $64.0 \pm 10.3$ \\
Height, cm & $170.7 \pm 6.7$ & $170.8 \pm 5.6$ \\
Body mass, kg & $68.0 \pm 8.2$ & $73.6 \pm 6.5$ \\
Mean WHO Oral Toxicity & $2.6 \pm 0.52$ & $3.0 \pm 0.0$ \\
Scale grade & & \\
Chemotherapy, $\mathrm{n}$ & 4 & 1 \\
Chemoradiotherapy, $\mathrm{n}$ & 6 & 4 \\
\hline
\end{tabular}

WHO, World Health Organization.

\section{Results}

Patient characteristics. The baseline characteristic of the patients are presented in Table II. There were no statistically significant differences between the groups at the baseline. It should be noted that no underweight patients were included in the present study. No patients required administration of opioid analgesics throughout the study.

Efficacy. The changes in efficacy outcomes during the study are listed in Table III. The WHO Oral Toxicity Scale grade had decreased by $41 \%$ in the ONCX group at $\sim 7$ days from the start of the study (Visit 2) compared with a minimal change in the control group. At the end of the study, the difference was even more prominent, with a 73 and $20 \%$ decrease from the baseline in the ONCX and control groups, respectively (Figs. 2 and 3).

A significant difference in number of days with regular food intake was observed. Over the entire testing period, patients in the ONCX group were able to eat normally during $65 \%$ of the days, in contrast to only $29 \%$ of the days in the control group (Fig. 4).

There were no statistically significant changes in absolute body mass between groups or even within each group, although there were clear positive and negative tendencies in the ONCX and control groups, respectively. Thus, mean change from the body mass baseline was calculated and it was found that ONCX caused a weight gain of $2.5 \mathrm{~kg}(-1.86$ to 6.86$)$ over 20 days, whereas the control group exhibited loss of $2.8 \mathrm{~kg}$ $(-4.96$ to -0.55$)(\mathrm{P}=0.03)$. No statistically relevant differences

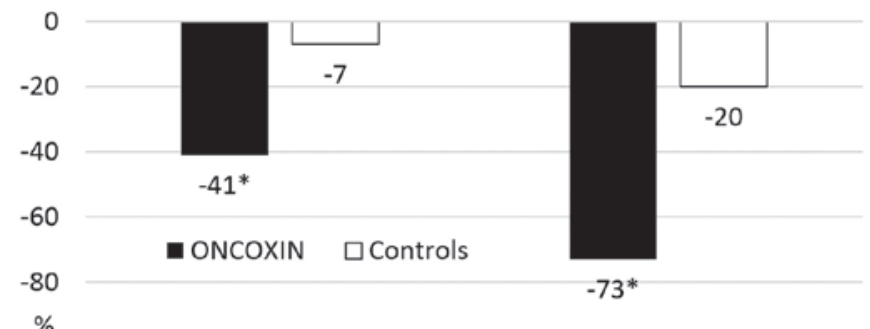

Figure 2. Changes in the WHO Oral Mucositis Scale grades during the study. Data are presented as $\%$ change from baseline. ${ }^{*} \mathrm{P}<0.05$ vs. controls.

in the number of days with normal appetite were observed. In the control group there were no days with normal appetite throughout the whole study, while in the ONCX group there were $4.80(0.59-9.01)$ days with normal appetite between Visits 2 and 3 ( $\mathrm{P}=0.13$ vs. controls).

Toxicity. In addition to efficacy outcomes, some differences in treatment toxicity were also observed (Table IV). The decrease of liver toxicity is of a special interest. The alanine aminotransferase and aspartate aminotransferase levels had decreased 4- to 7-fold at the end of the treatment in the ONCX group, whereas no changes were observed in the control group. The leukocyte count and incidence of infections were also in favour of the ONCX group. Therefore, ONCX was found to be well-tolerated, without associated adverse events.

\section{Discussion}

Different treatment options and drugs have been tested in OM, only some of which exhibited clinical relevance such as oral cryotherapy, keratinocyte growth factor and amino acids enriched diets. Therefore, there is a clear requirement for new approaches to the prevention and effective treatment of OM. ONCX is a multicomponent nutritional supplement that contains vitamins, amino acids, microelements and macromolecular biologically active substances. These substances have broad-spectrum antioxidant, anti-inflammatory and antimicrobial activities (6).

Glycine, cysteine, zinc, vitamin $\mathrm{C}$ and epigallocatechin gallate (EGCG) are potent antioxidants (6). EGCG is a naturally occurring polyphenol and the main component of green tea extract. A high effectiveness of EGCG in acute esophagitis induced by radiotherapy or chemoradiotherapy was reported in clinical trials $(7,8)$.

Another component of ONCX is glucosamine. As shown in clinical studies, glucosamine decreases the systemic levels 
Table III. Efficacy outcome changes during the study.

\begin{tabular}{|c|c|c|c|}
\hline \multirow[b]{2}{*}{ Efficacy outcomes } & \multicolumn{2}{|c|}{ Groups [mean (95\% confidence interval)] } & \multirow[b]{2}{*}{ P-value } \\
\hline & Oncoxin $(n=10)$ & Controls $(n=5)$ & \\
\hline \multicolumn{4}{|c|}{ Mean WHO Oral Toxicity Scale grade } \\
\hline Baseline & $2.60(2.23-2.97)$ & 3.0 & 0.25 \\
\hline Visit 2 & $1.56(0.88-2.23)$ & $2.80(2.24-3.36)$ & 0.02 \\
\hline Visit 3 & $0.70(0.35-1.05)$ & $2.40(1.72-3.08)$ & $<0.001$ \\
\hline \multicolumn{4}{|l|}{ Body mass } \\
\hline Baseline & $68.0(62.2-73.8)$ & $73.6(65.5-81.7)$ & 0.31 \\
\hline Visit 2 & $68.1(62.7-73.5)$ & $72.2(63.7-80.7)$ & 0.51 \\
\hline Visit 3 & $69.6(63.6-75.6)$ & $71.6(63.1-80.0)$ & 0.86 \\
\hline \multicolumn{4}{|c|}{ Days with intake of regular food } \\
\hline Visit 1-Visit 2 & $3.90(3.19-4.61)$ & $1.6(-0.66-3.86)$ & 0.03 \\
\hline Visit 2-Visit 3 & $9.70(5.78-13.62)$ & $4.4(-1.26-10.06)$ & 0.09 \\
\hline Visit 1-Visit 3 & $13.6(9.43-17.76)$ & $6.00(-1.90-13.90)$ & 0.04 \\
\hline
\end{tabular}

Bold print indicates statistical significance. WHO, World Health Organization.

Table IV. Individual toxicity grades according to grading criteria of the National Cancer Institute Common Toxicity Criteria (ver. 2).

Groups [mean (95\% confidence interval)]

\begin{tabular}{|c|c|c|c|}
\hline \multirow{2}{*}{ Toxicity measures } & & \multirow[b]{2}{*}{ P-value } \\
\hline & Oncoxin $(n=10)$ & Controls $(n=5)$ & \\
\hline \multicolumn{4}{|l|}{ Leukocytes } \\
\hline Baseline & $1.30(0.95-1.65)$ & 1.00 & 0.37 \\
\hline Visit 2 & $0.60(0.23-0.97)$ & 1.00 & 0.25 \\
\hline Visit 3 & $0.11(-0.15-0.37)$ & $0.80(0.24-1.36)$ & 0.04 \\
\hline \multicolumn{4}{|l|}{ Infections } \\
\hline Baseline & $1.70(1.35-2.05)$ & $1.80(1.24-2.36)$ & 0.77 \\
\hline Visit 2 & $1.00(0.66-1.34)$ & $1.40(0.29-2.51)$ & 0.31 \\
\hline Visit 3 & $0.11(-0.15-0.37)$ & $1.20(0.64-1.76)$ & $<0.005$ \\
\hline \multicolumn{4}{|c|}{ Alanine aminotransferase } \\
\hline Baseline & $1.50(0.99-2.01)$ & 1.00 & 0.13 \\
\hline Visit 2 & $0.70(0.22-1.18)$ & 1.00 & 0.37 \\
\hline Visit 3 & $0.33(-0.05-0.72)$ & 1.00 & 0.04 \\
\hline \multicolumn{4}{|c|}{ Aspartate aminotransferase } \\
\hline Baseline & $1.50(0.99-2.01)$ & 1.00 & 0.13 \\
\hline Visit 2 & $0.40(0.03-0.77)$ & 1.00 & 0.08 \\
\hline Visit 3 & $0.22(-0.12-0.56)$ & 1.00 & 0.012 \\
\hline
\end{tabular}

Bold print indicates statistical significance.

of C-reactive protein and prostaglandin E2 (9), whereas in murine models of colitis, glucosamine intake led to decreased expression of tumor necrosis factor (TNF)- $\alpha$ and interleukin-1 in the colonic mucosa, effects which were mediated by downregulation of nuclear factor (NF)- $\mathrm{B}$ (10). Dietary zinc also suppresses systemic inflammation, as shown by the substantial decrease in TNF- $\alpha$ production in elderly patients (11). Glycyrrhizinic acid (GA) is a well-known substance with hepatoprotective activity and broad-spectrum anti-inflammatory properties, mediated via NF- $\kappa \mathrm{B}$ and cyclooxygenase-2 (6). GA can markedly decrease liver damage in patients who receive the MMF chemotherapeutic regimen 
A

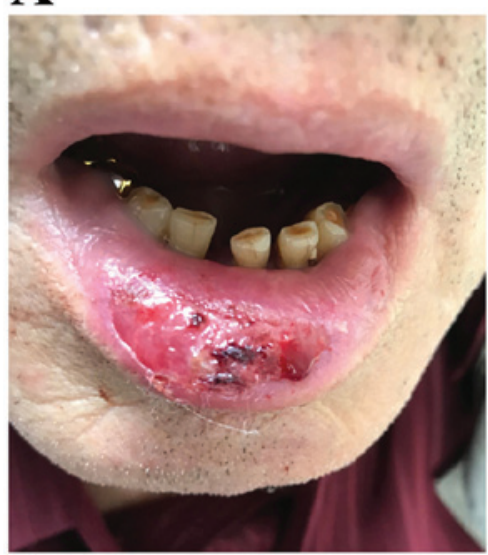

B

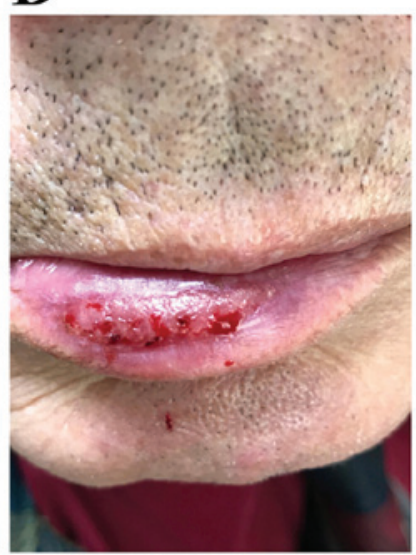

C

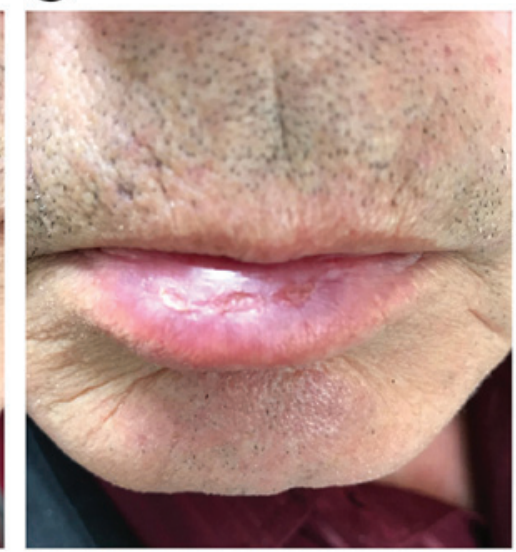

Figure 3. Clinical case. Male patient, $68 \mathrm{y} / \mathrm{o}$, with cancer of the tongue, treated with combined chemotherapy and radiotherapy. (A) Baseline, (B) visit 2 (7 days) and (C) visit 3 (20 days).

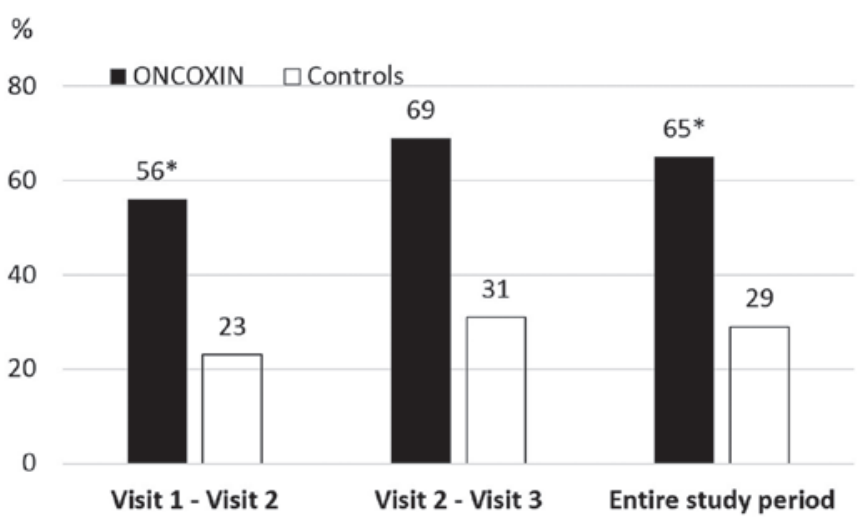

Figure 4. Percentage of days with intake of food during study intervals. ${ }^{*} \mathrm{P}<0.05$ compared with controls.

for breast cancer, or FOLFOX/XELOX for gastrointestinal tumors $(12,13)$. It is hypothesized that these effects of GA are due to its antioxidant and anti-inflammatory properties.

The antimicrobial properties of ONCX are also notable. Infectious complications of OM are common, and markedly contribute to clinical deterioration. Additional zinc intake is accompanied by decreased frequency of infections in elderly patients, as well as in children and adolescents with acute leukemia $(11,14)$.

Vitamins that regulate one-carbon metabolism pathway (pyridoxine, folate and cobalamin) play a crucial role in DNA structure stabilization and repair. Their deficiency is associated with single- and double-strand DNA breaks, which are similar to the effects of ionizing radiation (15).

Taken together, these properties may be responsible for the efficacy of ONCX in decreasing OM symptoms, and in improving the ability to ingest solid foods and maintain body weight. In addition, ONCX components help control infectious complications and decrease the toxicity of therapy with regards to leukocyte count and liver damage. The trend of improvement in body mass and appetite and the lack of statistically meaningful differences are hypothesized to be due to the insufficient number of patients included in the present study. Another possible reason is that the baseline body mass index of all included patients was $>19$, and there were no underweight patients, whereas some were overweight.

In conclusion, this was a pilot study conducted to demonstrate the benefits of ONCX nutritional supplement in OM. It was observed that ONCX rapidly improved the symptoms of OM, maintained body mass and decreased the toxicity of anticancer therapy. Although the number of participants was small, the results appear to be promising. However, further large-scale studies are required to confirm the value of ONCX in the management of OM.

\section{Acknowledgements}

Not applicable.

\section{Funding}

Catalysis S.L. provided Oncoxin nutritional supplement for the purposes of this study. No other funding was received.

\section{Availability of data and materials}

The datasets used and/or analyzed during the present study are available from the corresponding author on reasonable request.

\section{Authors' contributions}

AS was responsible for patient selection, treatment and interpretation of final results. EB was involved in study design, protocol and procedure development and interpretation of final results. ES was involved in study design, protocol and procedures development and manuscript preparation. FP performed statistical analysis and was a major contributor in writing the manuscript. All authors have read and approved the final version of this manuscript.

\section{Ethics approval and consent to participate}

This study was approved by the Ethics Committee of The Loginov Moscow Clinical Scientific Center, protocol 3/2017 4.17.17. The inclusion period was between November 2017 and March 2018. 


\section{Patient consent for publication}

In accordance with the Declaration of Helsinki, all patients provided written informed consent to participate in the study and to publish its results and anonymized photos.

\section{Competing interests}

Dr Eduardo Sanz is a Scientific Department Managing Director of Catalysis S.L., the manufacturer and provider of Oncoxin for the purposes of the study. All other authors declare that they have no competing interests to disclose.

\section{References}

1. Shumsky AV: Pathology of oral cavity induced by chemotherapy and radiotherapy in oncology patients. In: Oncology for Dentists. Stomatology for Oncologists. «Vse pravilno» (ed). Moscow, pp234-246, 2016.

2. Lalla RV, Sonis ST and Peterson DE: Management of oral mucositis in patients who have cancer. Dent Clin North Am 52: 61-77, viii, 2008.

3. Campos MI, Campos CN, Aarestrup FM and Aarestrup BJ: Oral mucositis in cancer treatment: Natural history, prevention and treatment. Mol Clin Oncol 2: 337-340, 2014.

4. Wardley AM, Jayson GC, Swindell R, Morgenstern GR, Chang J, Bloor R, Fraser CJ and Scarffe JH: Prospective evaluation of oral mucositis in patients receiving myeloablative conditioning regimens and haemopoietic progenitor rescue. $\mathrm{Br} \mathrm{J}$ Haematol 110: 292-299, 2000

5. Al-Mahtab M, Akbar SM, Khan MS and Rahman S: Increased survival of patients with end-stage hepatocellular carcinoma due to intake of ONCOXIN ${ }^{\circledR}$, a dietary supplement. Indian J Cancer 52: 443-446, 2015.

6. Dzhugashvili M, Pokrovsky VS and Snegovoy AV: Novel approaches for the correction of micronutrient deficiency in patients with malignant tumors. Malignant Tumours 2: 55-65, 2016.
7. Zhao H, Zhu W, Xie P, Li H, Zhang X, Sun X, Yu J and Xing L: A phase I study of concurrent chemotherapy and thoracic radiotherapy with oral epigallocatechin-3-gallate protection in patients with locally advanced stage III non-small-cell lung cancer. Radiother Oncol 110: 132-136, 2014.

8. Zhao H, Xie P, Li X, Zhu W, Sun X, Sun X, Chen X, Xing L and $\mathrm{Yu}$ J: A prospective phase II trial of EGCG in treatment of acute radiation-induced esophagitis for stage III lung cancer. Radiother Oncol 114: 351-356, 2015.

9. Kantor ED, Lampe JW, Navarro SL, Song X, Milne GL and White E: Associations between glucosamine and chondroitin supplement use and biomarkers of systemic inflammation. J Altern Complement Med 20: 479-485, 2014.

10. Bak YK, Lampe JW and Sung MK: Effects of dietary supplementation of glucosamine sulfate on intestinal inflammation in a mouse model of experimental colitis. J Gastroenterol Hepatol 29: 957-963, 2014.

11. Prasad AS, Beck FWJ, Bao B, Fitzgerald JT, Snell DC, Steinberg JD and Cardozo LJ: Zinc supplementation decreases incidence of infections in the elderly: Effect of zinc on generation of cytokines and oxidative stress. Am J Clin Nutr 85: 837-844, 2007.

12. Akimoto M, Kimura M, Sawano A, Iwasaki H, Nakajima Y, Matano S and Kasai M: Prevention of cancer chemotherapeutic agent-induced toxicity in postoperative breast cancer patients with glycyrrhizin (SNMC). Gan No Rinsho 32: 869-872, 1986 (In Japanese).

13. Yan Y, Mo Y and Zhang D: Magnesium isoglycyrrhizinate prevention of chemotherapy-induced liver damage during initial treatment of patients with gastrointestinal tumors. Zhonghua Gan Zang Bing Za Zhi 23: 204-208, 2015 (In Chinese).

14. Consolo LZ, Melnikov P, Cônsolo FZ, Nascimento VA and Pontes JC: Zinc supplementation in children and adolescents with acute leukemia. Eur J Clin Nutr 67: 1056-1059, 2013.

15. Ames BN: DNA damage from micronutrient deficiencies is likely to be a major cause of cancer. Mutat Res 475: 7-20, 2001.

(i) (9) This work is licensed under a Creative Commons Attribution-NonCommercial-NoDerivatives 4.0 International (CC BY-NC-ND 4.0) License. 